\title{
Research Progress of Combination of Traditional Chinese and Western Medicine in Treatment of Infertility in Older Women
}

\author{
Tingjuan Ren', Hongli Zhu²*, Rui Wang3 \\ ${ }^{1}$ Shaanxi University of Traditional Chinese Medicine, Xianyang 712046, Shaanxi Province, China \\ 2 The Affiliated Hospital of Shaanxi University of Traditional Chinese Medicine, Xianyang 712000, Shaanxi Province, China \\ 3 The Second Affiliated Hospital of Xi'an Jiaotong University, Xi'an 710004, Shaanxi Province, China
}

*Corresponding author: Hongli Zhu, 15909104459@163.com

\begin{abstract}
The main cause of infertility in elderly women is poor ovarian function, decreased quality and number of oocytes, which ultimately leads to poor fertility. Low ovarian function is the most difficult point in the research of reproductive medicine and reproductive health. At present, the treatment of integrated traditional Chinese and Western medicine has gradually achieved results. Western medicine mainly regulates endocrine and assisted reproductive treatment by supplementing estrogen and progesterone, while traditional Chinese medicine (TCM) mainly focuses on invigorating the kidney, adding and subtracting according to the syndrome. The treatment effect of TCM or western medicine alone is not significant. Many clinical observations show that integrated TCM and western medicine focuses on the specialties of western medicine, which not only makes up for the insufficiency of the slow action of TCM, but also improves its clinical efficacy when combined with western medicine. Based on domestic and foreign research, this article summarizes the treatment of infertility in elderly women with integrated TCM and western medicine.
\end{abstract}

Keywords: Senile infertility; Integrated traditional Chinese and western medicine; Research progress

Publication date: July 2021; Online publication: July 31, 2021

\section{Introduction}

Older-age women are defined as age $\geq 35$ years old. At present, with the increasing population of latemarriage and childbearing women, the problem of fertility at older ages has become increasingly prominent. Among older women, the incidence of infertility is positively correlated with age. Studies have shown that the incidence of infertility is $15 \%$ at the age of $30-35,30 \%$ at the age of $35-490$, and about $64 \%$ at the age of 40-45 ${ }^{[1]}$. In addition to the low number of follicles and the low quality of oocytes, the uterine muscle tumors, adenomyosis, and endometriosis are also the main causes of infertility in elderly women, because the incidence of uterine diseases such as uterine fibroids also increases with age. The "Guidelines for Clinical Practice of Assisted Reproduction in Advanced-age Infertile Women in China" pointed out: Women $\geq 35$ years of age have a significant increase in the risk of spontaneous miscarriage with age, a significant decrease in pregnancy rate and live birth rate, various pregnancy complications, complications, and birth defects in newborns. Therefore, in the treatment of infertility of elderly women, the ovarian preparation function must be evaluated first. If necessary, assisted reproductive technology can be used to shorten the time to pregnancy. In the treatment of infertility in elderly women, TCM mainly uses nourishing kidney and nourishing essence, and reconciling chong and ren as the main methods. Chinese medicine believes that the kidney stores essence and governs reproduction, and the "kidney-tiangui-chongren-uterine" 
axis plays a key role in promoting reproduction. "Suwen·Ancient Innocence Theory" says: "A woman is seven years old, her kidneys are strong, and her teeth are longer; on February 7th, when Tianguizhi reaches, Ren's pulse is strong, Taichong pulse is strong, and menstrual events are in the present day, so there are sons; The kidney Qi is equal, so the real tooth grows and grows extremely; at 47 strong muscles and bones, long hair, strong body; at 57, Yangming pulse decline, face begins to focus, originate fall; at 67, three Yang pulse declines. On the upper side, the face is all burnt, and the hair starts to be white; Qi, Ren pulse deficiency, Taichong pulse decay, Tiangui exhaustion, tunnel is not flowing, so the shape is bad and there is no child." "The Yellow Emperor's Internal Classic" says: "The kidney is the master of the sting, also the foundation of sealing, the essence." It fully shows that the occurrence of infertility in elderly women is particularly closely related to the kidney. The kidney governs reproduction, and the egg is the essence of the kidney. If the kidney essence is full, the follicle develops normally; the kidney qi is strong, the pulse is effective, and the Taichong pulse is the basic condition for the elimination of the egg ${ }^{[2]}$. Therefore, the treatment of infertility in elderly women is basically based on kidney supplementation, which can be added or subtracted with the disease.

\section{Etiology and pathogenesis}

The main causes of infertility in elderly women in western medicine are ovarian function decline and decreased oocyte quality. Some patients are likely to be accompanied by pelvic fallopian tube factors and male factors. In TCM, the onset of infertility in elderly women is related to insufficient kidney essence and imbalance of Chong and Ren, which are mainly based on kidney deficiency, stagnation of Qi, phlegm coagulation, and blood stasis as indicators.

\section{Treatment}

\subsection{TCM treatment}

Traditional Chinese medicine treats the infertility of elderly females with kidney supplementation. Professor Luo Zhijuan puts forward the "three-point method" for elderly female infertility patients, which will "tonify the kidney, regulate the week and assist pregnancy" throughout, and has achieved more significant clinical effects ${ }^{[3]}$. Some scholars use yin and yang and six channels dialectical stages to treat elderly female infertility, nourishing yin and yang during menstruation, warming the spleen to dispel cold and dampness, nourishing yin and nourishing Qi during follicular period, promoting Qi and activating blood during ovulation, dysmenorrhea, and nourishing liver and kidney during luteal phase. Regulate yin and yang. Staged medication to balance yin and yang can induce the development, maturation, and ovulation of dominant follicles, so that the patient's yin and yang can be secreted, and the patient can become pregnant successfully. Therefore, for elderly women with infertility, whether it is staged or typed treatment, nourishment of the kidney runs throughout.

\subsection{Western medicine treatment}

Western medicine treatment methods for elderly infertility patients mainly include hormone therapy and assisted reproductive technology. For female infertility patients $\geq 35$ years of age who are trying to conceive for six months without contraception, a comprehensive evaluation and treatment of infertility should be carried out, and ovarian function should be assessed at the same time. For elderly patients receiving in vitro fertilization (IVF) treatment, as the age increases, the cumulative pregnancy rate and live birth rate of IVF decrease, and the abortion rate increases. Ovulation induction is an important part of the in vitro fertilization - embryonic transfer (IVF-ET) process. Some scholars have found that the short-term, micro-stimulation, long-acting, long-acting, and antagonist programs have no effect on pregnancy rates and pregnancy outcomes. There is a significant difference, and the pregnancy rate of the micro stimulation program and 
the long-acting long-term program is higher than the other two groups, but there is no statistical significance. In clinical work, individualized selection of ovulation induction programs should be carried out according to specific conditions. Guidelines for the selection of IVF fertilization methods are recommended: Intraspermic sperm injection (ICSI) does not improve the outcome of ART in elderly women, and the choice of IVF or ICSI has nothing to do with age. Pre-embryo transfer genetic screening (PGS) is feasible for older women who are $>38$ years old or who have repeated implantation failures and repeated spontaneous abortions.

\subsection{Integrated traditional Chinese and western medicine treatment}

Some scholars have discovered through research that TCM, as an adjuvant therapy for ART, can not only improve the quality of oocytes and improve the outcome of pregnancy, it has unique advantages and has achieved significant effects. The application of TCM to assisted reproductive technology is a current research hotspot and the general trend in the future. Therefore, the application of TCM and acupuncture to assisted reproductive technology can further improve clinical efficacy.

\section{Conclusion}

At present, the incidence of infertility is gradually increasing. Late marriage and late childbearing have led to a gradual increase in the number of elderly women. Its effective and efficient treatment has become a current research hotspot. This article discusses the current status and treatment methods of infertility in elderly women. Both western medicine and TCM have their characteristics. Relevant clinical studies have shown that TCM can improve the ovarian function of elderly women with infertility, and at the same time can reduce the amount of gonadotropin ( $\mathrm{Gn}$ ) in patients during assisted reproductive technologies (ART). At the same time, studies have shown that TCM can also alleviate ovarian hyperstimulation in patients undergoing IVF-ET. In the adjuvant treatment of IVF-ET, the most frequently used TCM are Chinese medicines that nourish the kidney and nourish the essence, such as Cuscuta, Dipsacus, Eucommia, etc. Some patient studies have found that most patients in IVF have liver depression, and drugs such as Bupleurum and turmeric can be added to soothing the liver. According to clinical results TCM has a significant effect on elderly women, through the main line of invigorating the kidney, soothing the liver, invigorating the spleen, and removing dampness. In Western medicine, if the ovarian function is low, the ovarian response is low, and the self-pregnancy is not pregnant for more than one year, IVF is recommended. At present, the application of TCM in the IVF-ET cycle has a significant effect and provides for intractable diseases in assisted reproductive technology. Therefore, in terms of the current clinical effects, the treatment of infertility in elderly women with integrated TCM and Western medicine is more worthy of clinical promotion. However, there is still a lack of multi-center joint large-sample data analysis. We need to further carry out prospective large-sample randomized controlled experiments to bring good news to more patients in the future.

\section{Funding}

Shaanxi Province Social Development Science and Technology Project (2016SF-362)

\section{Disclosure statement}

The author declares no conflict of interest. 


\section{References}

[1] Jiang L, Chen Y, Luo X, et al., 2019, Clinical Practice Guidelines for Assisted Reproduction in Advanced-age Infertile Women in China. Chinese Journal of Evidence-Based Medicine, 19(03) :253-270.

[2] Han Xue, 2018, Clinical Observation of Bushen Zhuyunfang Combined with Clomiphene in the Treatment of Ovulatory Infertility Due to Kidney Deficiency in Elderly Women. Heilongjiang University of Traditional Chinese Medicine.

[3] Pan Y, Luo Z, Wu Y, et al., 2020, Professor Luo Zhijuan, a Famous Traditional Chinese Medicine Doctor in Guangxi, "Three-Point Method" Experience in the Treatment of Infertility in Elderly Women. Journal of Hainan Medical University, 26(13): 1033-1036. 